MATEMATIKA, 2017, Volume 33, Number 2, 191-206

(C) Penerbit UTM Press. All rights reserved

\title{
Fixed point method and its improvement for the system of Volterra-Fredholm integral equations of the second kind
}

\author{
${ }^{1}$ Talaat I. Hasan, ${ }^{2}$ Shaharuddin Salleh, ${ }^{3}$ Nejmaddin A. Sulaiman \\ 1,3 Department of Mathematics, Salahaddin University \\ 44002 SUH, Erbil, Kurdistan Region, Iraq \\ ${ }^{2}$ Department of Mathematical Sciences, University Technologi Malaysia \\ 81310 UTM Skudai, Johor, Malaysia \\ e-mail (corresponding author): ss@utm.my
}

\begin{abstract}
In this paper, we consider the system of Volterra-Fredholm integral equations of the second kind (SVFI-2). We proposed fixed point method (FPM) to solve SVFI-2 and improved fixed point method (IFPM) for solving the problem. In addition, a few theorems and two new algorithms are introduced. They are supported by numerical examples and simulations using Matlab. The results are reasonably good when compared with the exact solutions.
\end{abstract}

Keywords Fixed point method, improved fixed point method, contraction mapping,and system Volterra-Fredholm integral equations of the second kind.

AMS mathematics subject classification 45D05, 34A12

\section{Introduction}

Integral equations have been one of the principal instruments in many different fields of science like applied mathematics physics, biology and engineering [1, 2]. Also integral equations are encountered in numerous applications in various areas [3]. In addition, they arise naturally in applications, in many fields of mathematics, science and technology [4]. They have studied both at the theoretical and practicallevels [5].

Solution of integral equations by numerical methods have grown widely grown in the last 25 years. The methods of integral equation are strongly used for treating many problems in mathematical physics [6]. Integral equations have many advantages witnessed by the increasing frequency of the integral equations in the literature and in many areas because some problems have their mathematical representation appear directly [7]. The VolterraFredholm integral equations appear from parabolic boundary value problems [6]. Integral equation focused on the numerical method of solution [8].

Jerri [9] was discussed fixed point iteration method to solve linear Volterra integral equation. Sulaiman [10] used fixed point method (FPM) to solve linear Fredholm integral equation of the second kind. Hasan [11] solved a certain system of Fredholm integral equation of the first kind. In addition, Waz waz [12] solved a system of linear Volterra integral equations by FPM.

The proposed method FPM and IFPM are used for obtaining the approximate solution of system Volterra-Fredholm integral equations of the second kind. Illustrative examples will be included to demonstrate the validity and applicability of the presented techniques to highlight the signification of the FPM and improve fixed point method (IFPM). 


\section{Definitions}

Definition 1 [9] A function $f: R^{p} \rightarrow R^{p}$ is said to be Lipschtiz on $B \subseteq R^{p}$ with Lipschtiz constant $L>0$ if

$$
\|f(x)-f(y)\| \leq L\|x-y\|,
$$

for all $x, y \in B$ for some norm \|\| on $R^{p}$.

Definition 2 [13] A map $T$ is called contraction mapping on the interval $[a, b]$ if it satisfy the following conditions:

1. $U \in C[a, b] \rightarrow T(U) \in C[a, b]$.

2. $T \in \operatorname{Lip}[a, b]$, with Lipchitz constant, $0<L<1$.

Definition $3[12,13]$ Let $V$ and $W$ be vector spaces over a field $F$. A function $T: V \rightarrow W$ is called a linear transformation if it satisfies the following conditions:

1. $\forall A, B \in V, T(A+B)=T(A)+T(B)$.

2. $\forall A \in V$ and $r \in F, T(r A)=r T(A)$.

Definition $4[6,14]$ The integral equation

$$
y(s)=f(s)+\lambda \int_{a}^{s} k(s, t) y(t) d t+\lambda^{*} \int_{a}^{b} g(s, t) y(t) d t,
$$

is called a linear Volterra-Fredholm integral equation of the second kind, where the functions $k(s, t)$ and $g(s, t)$ are called kernels of integral equation, $0<\lambda<1$ and $0<\lambda^{*}<1$ such that $f(s), k(s, t)$ and $g(s, t)$ are known functions on $R=\{(s, t), a \leq t<s \leq b\}$ and $y(s)$ is unknown function.

Definition $5[12,15]$ A kernel $k(s, t)$ is said to be a symmetric kernel if $k(s, t)=k(t, s)$ for all $s, t \in R$.

\section{System of linear Volterra-Fredholm integral equations of the sec- ond kind}

The system of $p$ Volterra-Fredholm integral equations of the second kind (SVFI-2) is given as follows:

$$
y_{i}(s)=f_{i}(s)+\sum_{k=1}^{m} \lambda_{i k} \int_{a}^{s} k_{i k}(s, t) y_{k}(t) d t+\sum_{k=1}^{m} \lambda_{i k}^{*} \int_{a}^{b} k_{i k}^{*}(s, t) y_{k}(t) d t,
$$

for $i=1,2,3, \ldots, p . f_{i}(s)$ is a continuous function on $[a, b], k_{i k}(s, t)$ and $k_{i k}^{*}(s, t)$ denote the given continuous kernel functions on $R=\{(s, t), a \leq t<s \leq b\}$ while $y_{i}(s)$ is the unknown function to be determined.

\section{$4 \quad$ FPM solution for SVFI-2 with symmetric kernels}

The system of linear Volterra- Fredholm integral equation of the second kind given in (1), this system can be written as follows:

$$
Y_{i}=F_{i}+\sum_{k=1}^{m} \lambda_{i k} K_{i k} Y_{k}+\sum_{k=1}^{m} \lambda_{i k}^{*} K_{i k}^{*} Y_{k}
$$


Where $Y_{i}=y_{i}(s), F_{i}=f_{i}(s), K_{i k}=k_{i k}(s, t)$ and $K_{i k}^{*}=k_{i k}^{*}(s, t)$, first, we suppose that the initial solution $Y_{i}^{0}=F_{i}$, then the first approximation is

$$
Y_{i}^{1}=F_{i}+\sum_{k=1}^{m} \lambda_{i k} K_{i k} Y_{k}^{0}+\sum_{k=1}^{m} \lambda_{i k}^{*} K_{i k}^{*} Y_{k}^{0},
$$

For second approximation, substitute $Y_{i}^{1}$ into (3) we get

$$
Y_{i}^{2}=F_{i}+\sum_{k=1}^{m} \lambda_{i k} K_{i k} Y_{k}^{1}+\sum_{k=1}^{m} \lambda_{i k}^{*} K_{i k}^{*} Y_{k}^{1},
$$

In general, the fixed-point method can be written as

$$
Y_{i}^{r+1}=F_{i}+\sum_{k=1}^{m} \lambda_{i k} K_{i k} Y_{k}^{r}+\sum_{k=1}^{m} \lambda_{i k}^{*} K_{i k}^{*} Y_{k}^{r}, \text { for } r=1,2,3, \ldots, \max
$$

where max is the maximum number of iterations.

If the relationship defined in Equation (5) converges then

$$
\begin{gathered}
\lim _{n \rightarrow \infty}\left|Y_{i}-Y_{i}^{r}\right|=0, \\
Y_{i}^{r+1}=M\left(Y_{i}^{r}\right),
\end{gathered}
$$

with

$$
M\left(Y_{i}^{r}\right)=F_{i}+\sum_{k=0}^{m} \lambda_{i k} K_{i k} Y_{k}^{r}+\sum_{k=0}^{m} \lambda_{i k}^{*} K_{i k}^{*} Y_{k}^{r}
$$

where $Y_{k}=y_{k}(s), Y_{i}^{r}=y_{k}^{r}(s)$.

The condition for the convergence property of the FPM is

$$
\left|\lambda_{i k}\right|<\frac{1}{M_{i k}}
$$

where

$$
M_{i k}=\sqrt{\int_{a}^{b} \int_{a}^{b}\left[k_{i k}(s, t)\right]^{2} d s d t} \text { and }\left|\lambda_{i k}^{*}\right|<\frac{1}{M_{i k}^{*}} \text { where } M_{i k}^{*}=\sqrt{\int_{a}^{b} \int_{a}^{b}\left[k_{i k}^{*}(s, t)\right]^{2} d s d t}
$$

\section{Algorithm for FPM}

Step 1 Let $Y_{i}^{0}=F_{i}$, for $i=1,2,3, \ldots p$ from equation (1).

Step 2 Find $Y_{i}^{r+1}$ from Equation (7), $r=1,2,3, \ldots, \max$.

Step 3 Compute the value of absolute error given by $e_{i}^{r}=\left|y_{i}(s)-y_{i}^{r}(s)\right|$.

Step 4 The approximate solutions converges if

$$
\lim _{r \rightarrow \infty}\left|y_{i}(s)-y_{i}^{r}(s)\right|=\varepsilon,
$$

where $\varepsilon$ is a value close to 0 . 
Example 1 Consider the following system

$$
\begin{aligned}
& y_{1}(s)=2 \sin (s)-\int_{0}^{s} y_{2}(t) d t \\
& y_{2}(s)=\cos (s)-0.4597+\int_{0}^{1} y_{1}(t) d t,
\end{aligned}
$$

with the exact solutions $y_{1}(s)=\sin (s)$ and $y_{2}(s)=\cos (s)$.

Solution. By applying FPM and its program, we obtain the approximate solutions of $y_{1}(s)$ and $y_{2}(s)$ as follows and shown in Table 1 .

$$
\begin{aligned}
& y_{1}^{0}(s)=2 \sin (s) \\
& y_{2}^{0}(s)=\cos (s)-0.4597 \\
& y_{1}^{1}(s)=\sin (s)+(0.4597) s \\
& y_{2}^{1}(s)=\cos (s)+0.4596 \\
& y_{1}^{2}(s)=\sin (s)-(0.4597) s \\
& y_{2}^{2}(s)=\cos (s)+0.2298 \\
& y_{1}^{3}(s)=\sin (s)-(0.2298) s \\
& y_{2}^{3}(s)=\cos (s)-0.2298
\end{aligned}
$$

\begin{tabular}{|c|c|c|c|c|c|c|c|}
\hline$s$ & $r$ & $\begin{array}{l}\text { Exact } \\
\text { solution of } \\
y_{1}(s)=\sin (s)\end{array}$ & $\begin{array}{l}\text { Approximate } \\
\text { values of } \\
y_{1}(s)\end{array}$ & $\begin{array}{l}\text { Absolute error } \\
e_{1}^{r}= \\
\left|y_{1}(s)-{ }^{*} y_{1}^{r}(s)\right|\end{array}$ & $\begin{array}{l}\text { Exact } \\
\text { solution of } \\
y_{2}(s)=\cos (s)\end{array}$ & $\begin{array}{l}\text { Approximate } \\
\text { values of } \\
y_{2}(s)\end{array}$ & $\begin{array}{l}\text { Absolute error } \\
e_{2}^{r}= \\
\mid y_{2}(s)-{ }^{*} y_{2}^{r}(s)\end{array}$ \\
\hline \multirow{11}{*}{0.2} & 0 & 0.19866933 & 0.39733866 & $1.1986 \times 10^{-1}$ & 0.98006657 & 0.52036888 & $4.5969 \times 10^{-1}$ \\
\hline & 2 & & 0.24463910 & $4.5969 \times 10^{-2}$ & & 1.03752878 & $5.7462 \times 10^{-2}$ \\
\hline & 4 & & 0.19292310 & $5.7462 \times 10^{-3}$ & & 0.97288380 & $7.1827 \times 10^{-3}$ \\
\hline & 6 & & 0.19938760 & $7.1827 \times 10^{-4}$ & & 0.98096442 & $8.9784 \times 10^{-4}$ \\
\hline & 8 & & 0.19866812 & $2.1212 \times 10^{-5}$ & & 0.98084223 & $2.2346 \times 10^{-4}$ \\
\hline & 10 & & 0.19866501 & $4.3245 \times 10^{-6}$ & & 0.98009944 & $3.3134 \times 10^{-5}$ \\
\hline & 12 & & 0.19866998 & $6.2156 \times 10^{-7}$ & & 0.98006933 & $3.2355 \times 10^{-6}$ \\
\hline & 14 & & 0.19866945 & $1.2678 \times 10^{-7}$ & & 0.98006600 & $5.7762 \times 10^{-7}$ \\
\hline & 16 & & 0.19866939 & $6.7693 \times 10^{-8}$ & & 0.98006679 & $2.2759 \times 10^{-7}$ \\
\hline & 18 & & 0.19866933 & $8.4678 \times 10^{-9}$ & & 0.98006657 & $4.6793 \times 10^{-8}$ \\
\hline & 20 & & 0.19866933 & $2.4468 \times 10^{-9}$ & & 0.98006657 & $5.6784 \times 10^{-9}$ \\
\hline
\end{tabular}

Table 1: FPM results for example 1 and compared to the exact solution

Figures 1(a) and 1(b) show a comparison between the exact and the approximate solutions, given in Example 1.

Example 2 Consider the following system

$$
\begin{aligned}
& y_{1}(s)=1+s+\frac{1}{2} \int_{0}^{s} y_{2}(t) d t-\int_{0}^{1}(s t) y_{1}(t) d t, \\
& y_{2}(s)=2+s+\int_{0}^{s} y_{1}(t) d t-\int_{0}^{1}(s t) y_{2}(t) d t,
\end{aligned}
$$

with the exact solutions $y_{1}(s)=e^{s}$ and $y_{2}(s)=2 e^{s}$. 


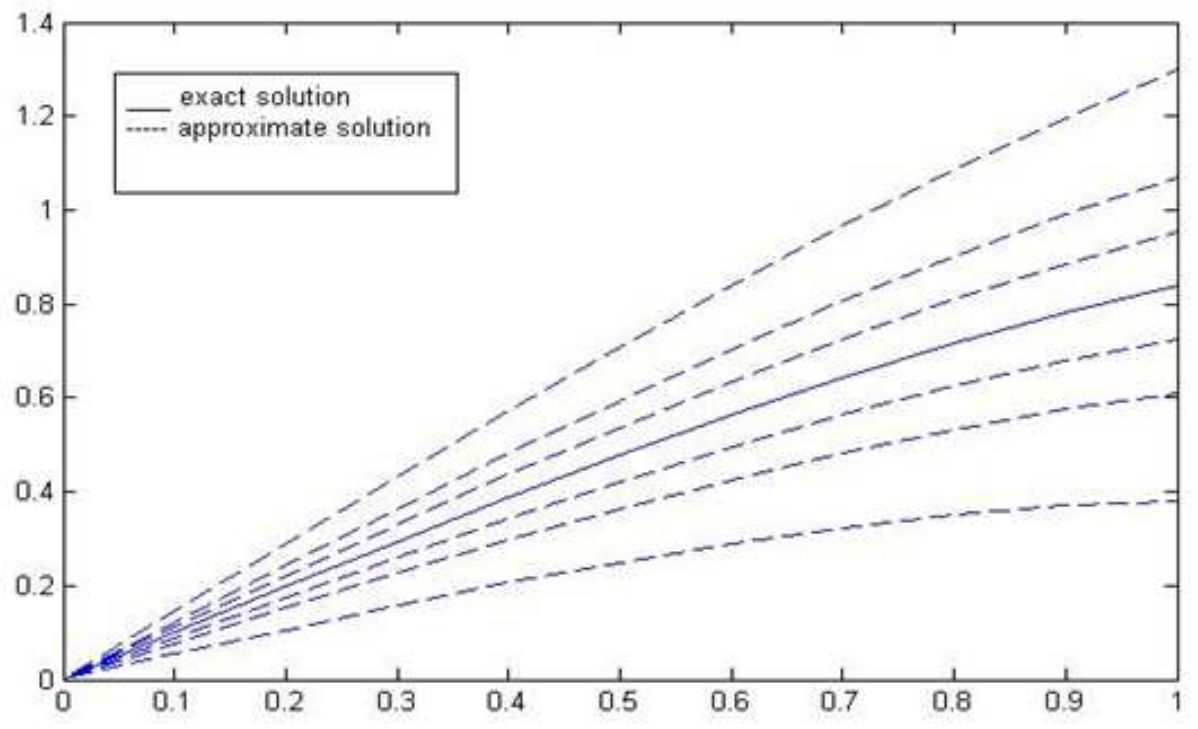

Figure 1(a): Graph $y_{1}(s)=\sin (s)$

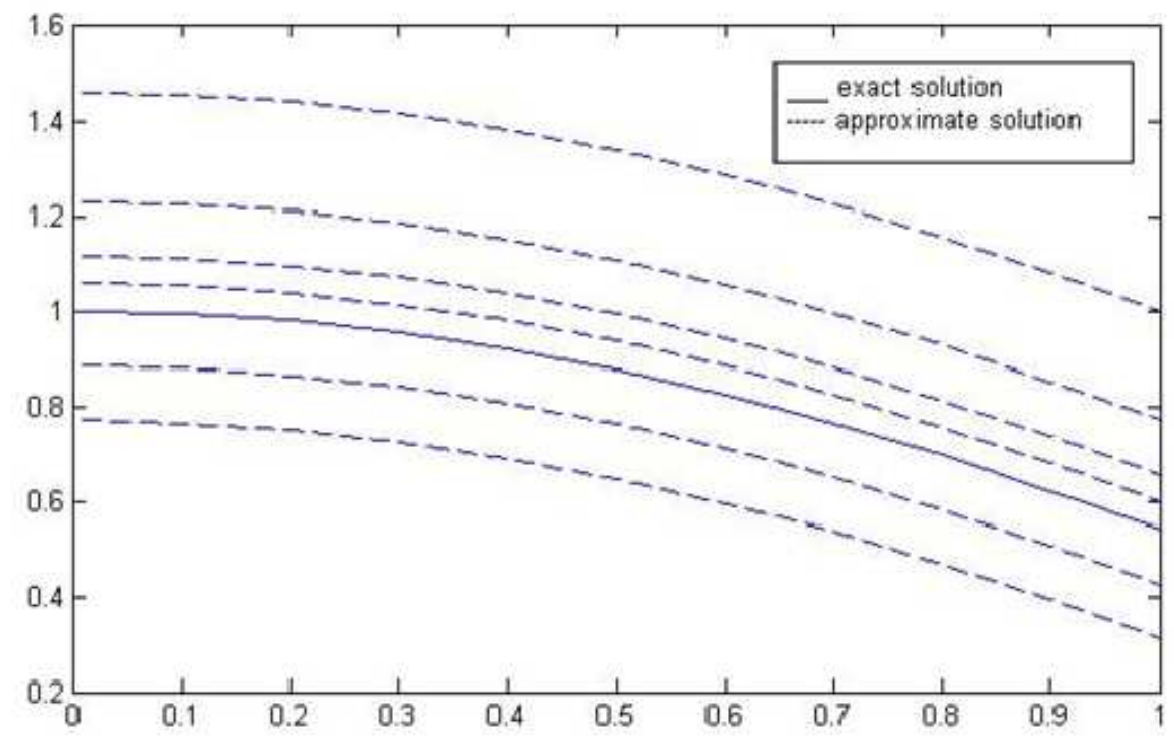

Figure 1(b): Graph $y_{2}(s)=\cos (s)$ 
Solution By applying FPM and its program, we obtain the approximate solutions of $y_{1}(s)$ and $y_{2}(s)$ as follows and shown in Table 2 .

$$
\begin{aligned}
& y_{1}^{0}(s)=1+s \\
& y_{2}^{0}(s)=2+s \\
& y_{1}^{1}(s)=1+1.1667 s+0.25 s^{2} \\
& y_{2}^{1}(s)=2+(2.1867) s+(0.5) s^{2} \\
& y_{1}^{2}(s)=1+(1.2153) s+(0.5417) s^{2}+(0.0833) s^{3} \\
& y_{2}^{2}(s)=2+(2.0856) s+(2.1667) s^{2}+(0.5) s^{3} \\
& y_{1}^{3}(s)=1+(0.9984) s+(0.0120) s^{2}+(0.3611) s^{3}+(0.0625) s^{4} \\
& y_{2}^{3}(s)=2+(1.9981) s+(1.0243) s^{2}+(0.6667) s^{3}+(0.1250) s^{4}
\end{aligned}
$$

\begin{tabular}{|c|c|c|c|c|c|c|c|}
\hline$s$ & $r$ & $\begin{array}{l}\text { Exact } \\
\text { solution of } \\
y_{1}(s)=e^{s}\end{array}$ & $\begin{array}{l}\text { Approximate } \\
\text { value of } \\
y_{1}(s)\end{array}$ & $\begin{array}{l}\text { Absolute error } \\
e_{1}^{r}= \\
\left|y_{1}(s)-y_{1}^{r}(s)\right|\end{array}$ & $\begin{array}{l}\text { Exact } \\
\text { solution of } \\
y_{1}(s)=2 e^{s}\end{array}$ & $\begin{array}{l}\text { Approximate } \\
\text { value of } \\
y_{2}(s)\end{array}$ & $\begin{array}{l}\text { Absolute error } \\
e_{2}^{r}= \\
\mid y_{2}(s)-y_{2}^{r}(s)\end{array}$ \\
\hline \multirow{11}{*}{0.2} & 0 & 1.22140275 & 1.20000000 & $2.1402 \times 10^{-2}$ & 2.44280551 & 2.20000000 & $2.4280 \times 10^{-1}$ \\
\hline & 2 & & 1.23205555 & $1.0652 \times 10^{-2}$ & & 2.45438888 & $1.1583 \times 10^{-2}$ \\
\hline & 4 & & 1.22134014 & $6.2613 \times 10^{-5}$ & & 2.44276323 & $4.2278 \times 10^{-5}$ \\
\hline & 6 & & 1.22140196 & $7.9443 \times 10^{-6}$ & & 2.44280090 & $4.6111 \times 10^{-6}$ \\
\hline & 8 & & 1.22140331 & $5.6095 \times 10^{-7}$ & & 2.44280607 & $5.5970 \times 10^{-7}$ \\
\hline & 10 & & 1.22140276 & $5.8513 \times 10^{-8}$ & & 2.44280552 & $5.0802 \times 10^{-9}$ \\
\hline & 12 & & 1.22140275 & $1.5373 \times 10^{-10}$ & & 2.44280551 & $6.2804 \times 10^{-10}$ \\
\hline & 14 & & 1.22140275 & $3.5835 \times 10^{-11}$ & & 2.44280551 & $3.2524 \times 10^{-11}$ \\
\hline & 16 & & 1.22140275 & $8.5912 \times 10^{-12}$ & & 2.44280551 & $7.7321 \times 10^{-13}$ \\
\hline & 18 & & 1.22140275 & $3.0213 \times 10^{-13}$ & & 2.44280551 & $1.6325 \times 10^{-14}$ \\
\hline & 20 & & 1.22140275 & $3.4421 \times 10^{-14}$ & & 2.44280551 & $2.5534 \times 10^{-15}$ \\
\hline
\end{tabular}

Table 2: FPM results for example 2 and compared to the exact solution

Figures 2(a) and 2(b) show a comparison between the exact and the approximate solutions, given in Example 2.

\section{IFPM to solve SVFI-2 with symmetric kernels}

Improved fixed-point method is obtained by adding $\alpha_{i} Y_{i}$ in both sides of Equation (7) where $\alpha_{i} \neq-1$ :

$$
\left(1+\alpha_{i}\right) Y_{i}=\alpha_{i} Y_{i}+M\left(Y_{i}\right)
$$

we get

$$
Y_{i}=\frac{1}{1+\alpha_{i}} M\left(Y_{i}\right)+\frac{\alpha_{i}}{1+\alpha_{i}} Y_{i}=M_{\alpha_{i}}\left(Y_{i}\right) .
$$

If ${ }^{*} Y_{i}$ verifies Equation (7) then it also verifies Equation (10). This means ${ }^{*} Y_{i}$ is a solution of Equation (5) expressed in the iterative form, as follows:

$$
{ }^{*} Y_{i}^{r+1}=M_{\alpha_{i}}\left({ }^{*} Y_{i}^{r}\right),
$$




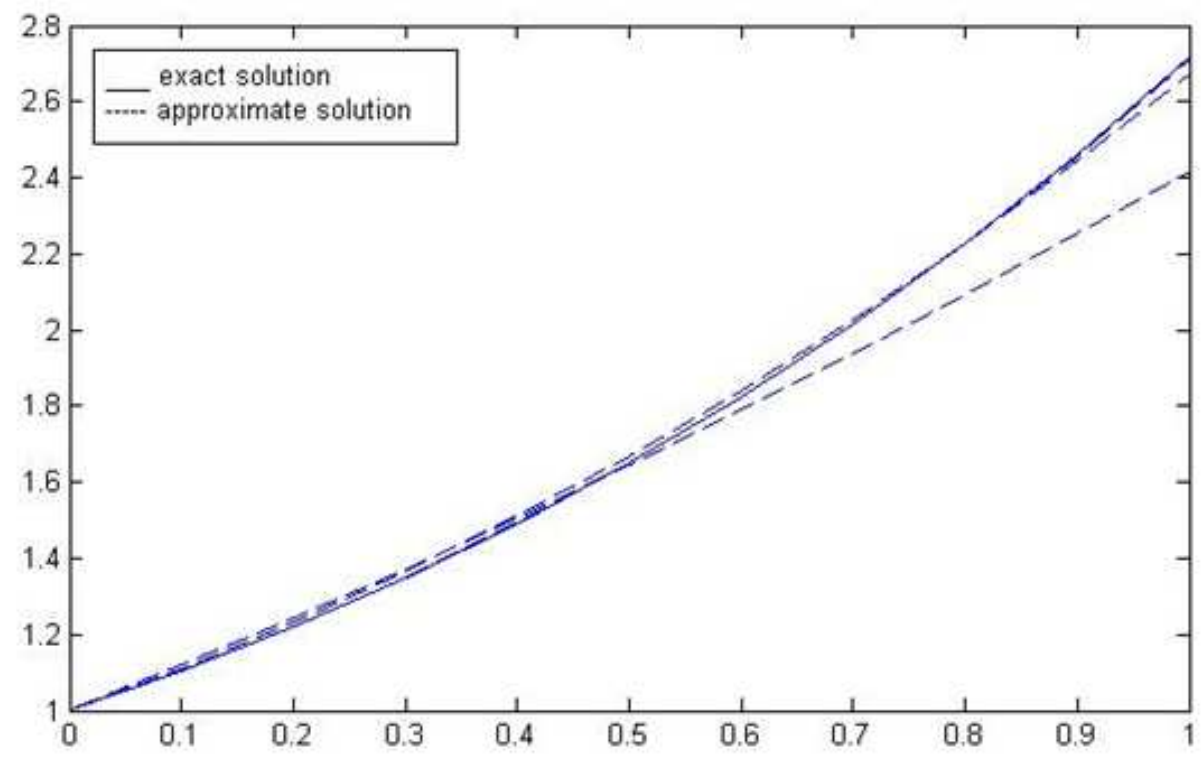

Figure 2(a): Graph $y_{1}(s)=e^{s}$

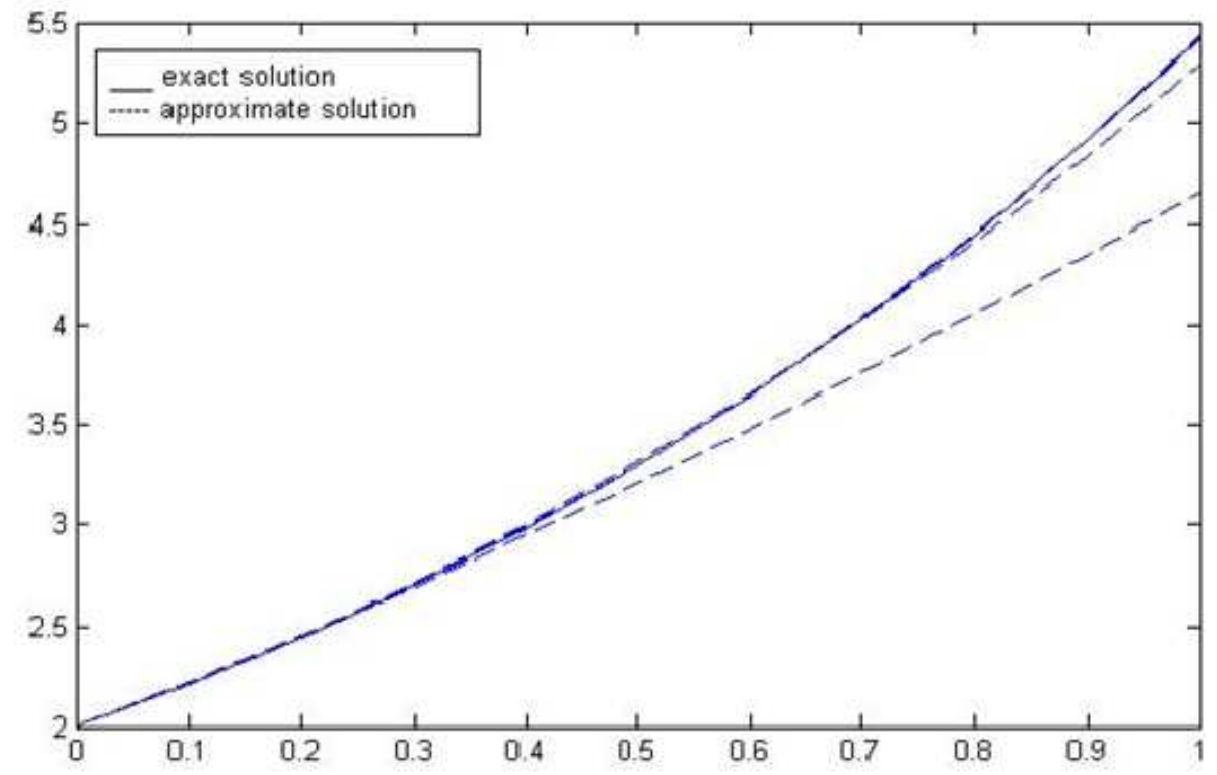

Figure 2(b): Graph $y_{2}(s)=2 e^{s}$ 
The approximate solutions in Equation (11) should converge faster than the iterative form defined in Equation (7) to the exact solution of Equation (1).

The selection for the optimal values of the scalar $\alpha_{i}$ is based on the following role

$$
\alpha_{i}=\frac{\left(\nu_{i}+\rho_{i}\right)}{2}
$$

where

$$
\nu_{i}=\sup _{[a, b]}\left\{M\left(Y_{i}\right)-M\left(Y_{i-1}\right)\right\} \text { and } \rho_{i}=\inf _{[a, b]}\left\{M\left(Y_{i}\right)-M\left(Y_{i-1}\right)\right\}
$$

\section{Algorithm for IFPM}

Step 1 Find the optimal values of the scalar $\alpha_{i}$ in Equations (12) and (13).

Step 2 Let $Y_{i}^{0}=F_{i}$, for $i=1,2,3, \ldots, p$, from Equation (1) which is the initial solution. Step 3 Adding $\alpha_{i} Y_{i}$ to both sides of Equation (7).

Step 4 Compute ${ }^{*} Y_{i}^{r+1}$ From Equation (11).

Step 5 Calculate $e_{i}^{r}=\left|y_{i}(s)-{ }^{*} y_{i}^{r}(s)\right|$.

Step 6 The approximate solutions converges faster if $\lim _{r \rightarrow \infty}\left|y_{i}(s)-y_{i}^{r}(s)\right|=\varepsilon$.

\section{Numerical examples about SVFI-2 and results by using IFPM}

The method of section 5 is very useful for finding the numerical solutions of SVFI-2. The computations associated with the examples were performed using MATLAB version 12.

Example 3 Find approximate solution ofa SVFI-2, in example 1 by using IFPM.

Solution Applying the numerical technique which is IFPM, we obtained the results for approximate solutions of $y_{1}(s)$ and $y_{2}(s)$, where the optimal values are $\alpha_{1}=-0.190$ and $\alpha_{2}=1.4597$, as shown in Table 3 .

$$
\begin{aligned}
& y_{1}^{0}(s)=2 \sin (s) \\
& y_{2}^{0}(s)=\cos (s)-0.4597 \\
& y_{1}^{1}(s)=(0.7654) \sin (s)+(0.5675) s \\
& y_{2}^{1}(s)=\cos (s)-0.0859 \\
& y_{1}^{2}(s)=(1.0550) \sin (s)-(0.0271) s \\
& y_{2}^{2}(s)=\cos (s)+0.0205 \\
& y_{1}^{3}(s)=(0.9871) \sin (s)-(0.0190) s \\
& y_{2}^{3}(s)=\cos (s)+0.0170
\end{aligned}
$$

Figures 3(a) and 3(b) show a comparison between the exact and the approximate solutions, given in Example 3.

Example 4 Find approximate solution ofa SVFI-2 in example 2 by using IFPM.

Solution Applying the numerical technique which is IFPM we obtained the results for approximate solutions of $y_{1}(s)$ and $y_{2}(s)$, where the optimal values are $\alpha_{1}=0.128$ and 
Table 3: IFPM results for example 3 and compared to the exact solution

\begin{tabular}{|c|c|c|c|c|c|c|c|}
\hline$s$ & $r$ & $\begin{array}{l}\text { Exact } \\
\text { solution of } \\
y_{1}(s)=\sin (s)\end{array}$ & $\begin{array}{l}\text { Approximate } \\
\text { Values of } \\
y_{1}(s)\end{array}$ & $\begin{array}{l}\text { Absolute error } \\
e_{1}^{r}= \\
\left|y_{1}(s)-{ }^{*} y_{1}^{r}(s)\right|\end{array}$ & $\begin{array}{l}\text { Exact } \\
\text { solution of } \\
y_{2}(s)=\cos (s)\end{array}$ & $\begin{array}{l}\text { Approximate } \\
\text { Values of } \\
y_{2}(s)\end{array}$ & $\begin{array}{l}\text { Absolute error } \\
e_{2}^{r}= \\
\left|y_{2}(s)-{ }^{*} y_{2}^{r}(s)\right|\end{array}$ \\
\hline & 0 & 0.19866933 & 0.39733866 & $1.9866 \times 10^{-1}$ & 0.98006657 & 0.52036888 & $4.5969 \times 10^{-1}$ \\
\hline \multirow[t]{10}{*}{0.2} & 2 & & 0.20418913 & $5.5198 \times 10^{-3}$ & & 1.00060762 & $2.0541 \times 10^{-2}$ \\
\hline & 4 & & 0.19597184 & $2.6974 \times 10^{-3}$ & & 0.98386228 & $3.7957 \times 10^{-3}$ \\
\hline & 6 & & 0.19887275 & $2.0342 \times 10^{-4}$ & & 0.97945051 & $6.1606 \times 10^{-4}$ \\
\hline & 8 & & 0.19868480 & $1.3549 \times 10^{-5}$ & & 0.98007752 & $1.0946 \times 10^{-5}$ \\
\hline & 10 & & 0.19866538 & $3.9434 \times 10^{-6}$ & & 0.98007323 & $6.6574 \times 10^{-6}$ \\
\hline & 12 & & 0.19866951 & $1.8462 \times 10^{-7}$ & & 0.98006581 & $7.6728 \times 10^{-7}$ \\
\hline & 14 & & 0.19866936 & $1.0472 \times 10^{-8}$ & & 0.98006656 & $1.0471 \times 10^{-8}$ \\
\hline & 16 & & 0.19866932 & $5.3205 \times 10^{-9}$ & & 0.98006658 & $1.0574 \times 10^{-9}$ \\
\hline & 18 & & 0.19866933 & $1.0570 \times 10^{-10}$ & & 0.98006657 & $8.7082 \times 10^{-10}$ \\
\hline & 20 & & 0.19866933 & $5.6451 \times 10^{-11}$ & & 0.98006657 & $4.9588 \times 10^{-11}$ \\
\hline
\end{tabular}

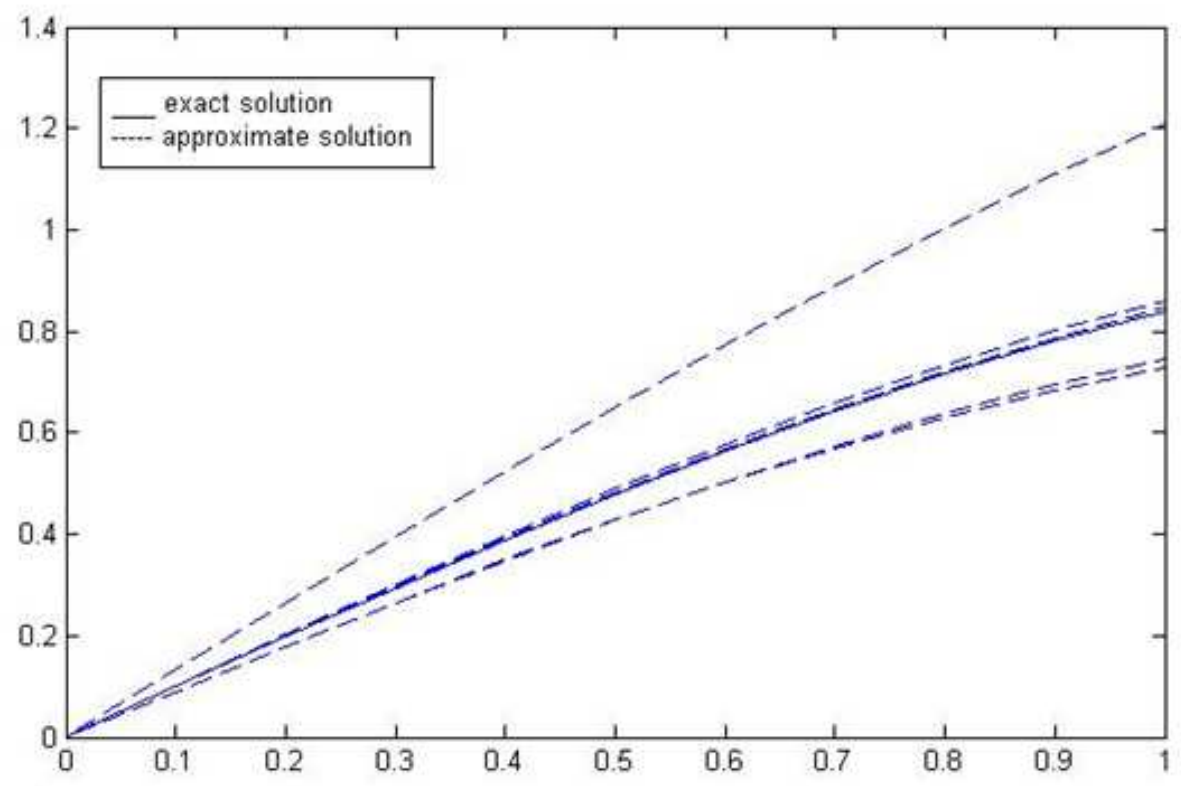

Figigure 3(a): Graph $y_{1}(s)=\sin (s)$ 


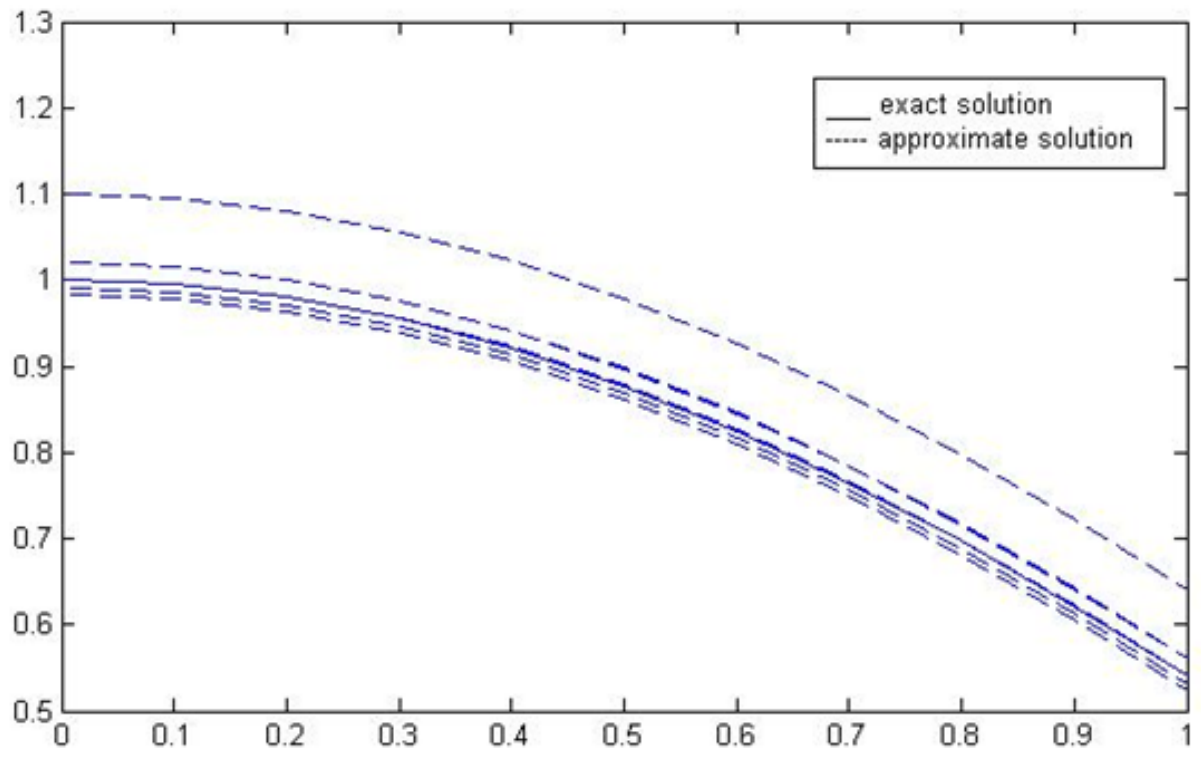

Figure 3(b): Graph $y_{2}(s)=\cos (s)$

$\alpha_{2}=0.315$, as shown in Table 4 .

$$
\begin{aligned}
& y_{1}^{0}(s)=1+s \\
& y_{2}^{0}(s)=2+s \\
& y_{1}^{1}(s)=1+(1.1478) s+(0.2216) s^{2} \\
& y_{2}^{1}(s)=12+(1.8872) s+(0.3802) s^{2} \\
& y_{1}^{2}(s)=1+(1.0717) s+(0.4434) s^{2}+(0.0562) s^{3} \\
& y_{2}^{2}(s)=2+(2.0201) s+(0.8086) s^{2}+(0.0964) s^{3} \\
& y_{1}^{3}(s)=1+(1.0265) s+(0.4980) s^{2}+(0.1259) s^{3}+(0.0107) s^{4} \\
& y_{2}^{3}(s)=2+(2.0204) s+(0.9618) s^{2}+(0.2281) s^{3}+(0.0183) s^{4}
\end{aligned}
$$

Figures 4(a) and 4(b) show a comparison between the exact and the approximate solutions, given in Example 4.

\section{$7 \quad$ Fixed point and contractive mapping}

The original iterative method is proposed as follows:

$$
Y_{i}^{r+1}(s)=F_{i}(s)+\sum_{k=0}^{m} \lambda K_{i k} Y_{i}^{r}+\sum_{k=0}^{m} \lambda^{*} K_{i k}^{*} Y_{i}^{r}, \text { for } r=0,1,2, \ldots, \max
$$


Table 4: IFPM results for example 4 and results compared to the exact solution

\begin{tabular}{|c|c|c|c|c|c|c|c|}
\hline$s$ & $r$ & $\begin{array}{l}\text { Exact } \\
\text { solution of } \\
y_{1}(s)=e^{s}\end{array}$ & $\begin{array}{l}\text { Approximate } \\
\text { Value of } \\
y_{1}(s)\end{array}$ & $\begin{array}{l}\text { Absolute error } \\
e_{1}^{r}= \\
\left|y_{1}(s)-{ }^{*} y_{1}^{r}(s)\right|\end{array}$ & $\begin{array}{l}\text { Exact } \\
\text { solution of } \\
y_{1}(s)=2 e^{s}\end{array}$ & $\begin{array}{l}\text { Approximate } \\
\text { Value of } \\
y_{2}(s)\end{array}$ & $\begin{array}{l}\text { Absolute error } \\
e_{2}^{r}= \\
\left|y_{2}(s)-{ }^{*} y_{2}^{r}(s)\right|\end{array}$ \\
\hline \multirow{11}{*}{0.2} & 0 & 1.22140275 & 1.20000000 & $2.1402 \times 10^{-2}$ & 2.44280551 & 2.20000000 & $2.4280 \times 10^{-2}$ \\
\hline & 2 & & 1.22163981 & $2.3705 \times 10^{-4}$ & & 2.44360370 & $7.9818 \times 10^{-3}$ \\
\hline & 4 & & 1.22132818 & $7.4575 \times 10^{-5}$ & & 2.44272708 & $7.8007 \times 10^{-5}$ \\
\hline & 6 & & 1.22140268 & $7.7778 \times 10^{-7}$ & & 2.44280553 & $1.7745 \times 10^{-7}$ \\
\hline & 8 & & 1.22140275 & $4.8578 \times 10^{-9}$ & & 2.44280551 & $2.3335 \times 10^{-8}$ \\
\hline & 10 & & 1.22140275 & $4.3890 \times 10^{-10}$ & & 2.44280551 & $4.1740 \times 10^{-9}$ \\
\hline & 12 & & 1.22140275 & $7.2264 \times 10^{-11}$ & & 2.44280551 & $6.8422 \times 10^{-11}$ \\
\hline & 14 & & 1.22140272 & $2.3652 \times 10^{-12}$ & & 2.44280551 & $6.2924 \times 10^{-13}$ \\
\hline & 16 & & 1.22140275 & $3.0622 \times 10^{-13}$ & & 2.44280551 & $3.0642 \times 10^{-14}$ \\
\hline & 18 & & 1.22140225 & $9.2433 \times 10^{-15}$ & & 2.44280551 & $8.2420 \times 10^{-15}$ \\
\hline & 20 & & 1.22140275 & $1.6952 \times 10^{-17}$ & & 2.44280551 & $1.8732 \times 10^{-17}$ \\
\hline
\end{tabular}

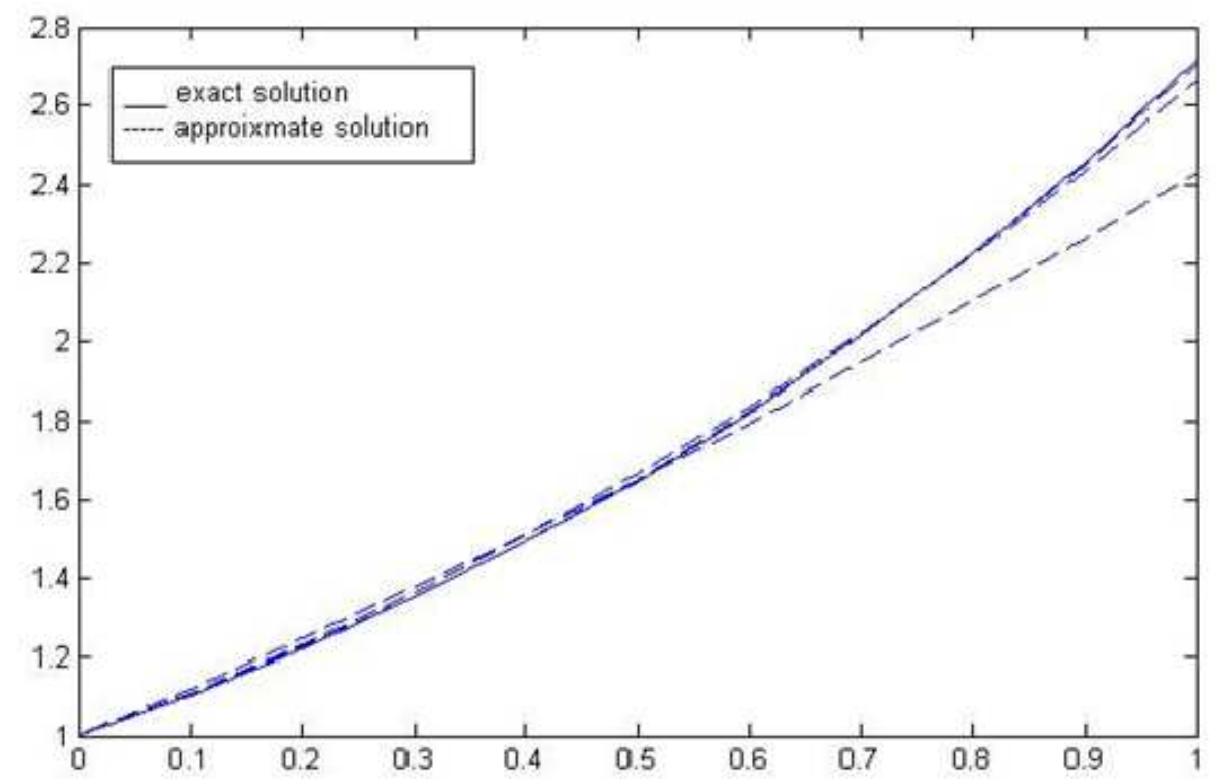

Figure 4(a): Graph $y_{1}(s)=e^{s}$ 


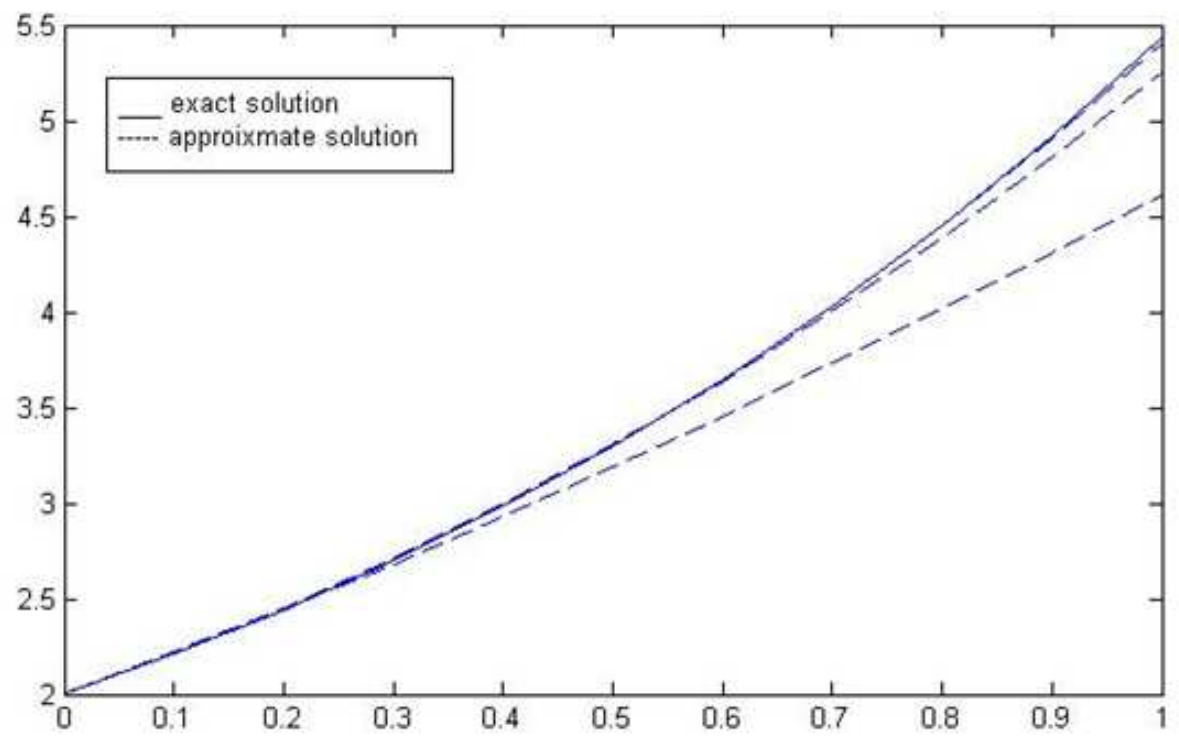

Figure 4(b): Graph $y_{2}(s)=2 e^{s}$

The approach of the sequences $Y_{i}^{r+1}(s)$ to $Y_{i}(s)$ has been studied for solving Equation (1). Let

$$
T\left(Y_{i}^{r}\right)=F_{i}(s)+\sum_{k=0}^{m} \lambda K_{i k} Y_{i}^{r}+\sum_{k=0}^{m} \lambda^{*} K_{i k}^{*} Y_{i}^{r} .
$$

Our solution in this work has been directed toward solving Equation (1). The integral equation (15) is searched in the following manner, the right hand side is supposed as a transformation $T$ on $Y_{i}^{r}$ expressed by $T\left(Y_{i}^{r}\right)$, when the left hand side denotes that the transformation had left this element $Y_{i}^{r+1}$ unchanged. That is

$$
Y_{i}^{r+1}=T\left(Y_{i}^{r}\right)
$$

which mean that the solution $Y_{i}^{r}$ which we search for the integral equation (15) expresses an especial values in the domain of the transformation $T$, called that which remains unaltered of stable under the transformation $T$. Each element $Y_{i}^{r}$ as defined in equation (16) is known a fixed point of the transformation $T$.

Lemma 1 [12] If a map $T$ satisfies the Lipchitz condition on the interval $[a, b]$ then there exists a positive constant $L$, such that $|T(y)-T(w)| \leq|y-w| L$, for all values $y, w \in R$. The constant $L$ is called Lipchitz constant.

Theorem 2 [3] Every contraction mapping is a Lipschitz function on $[a, b]$.

Theorem 3 [3] Every Lipschitz function is a continuous function on $[a, b]$.

Theorem 4 Let $T$ be a contraction mapping on $R$ then:

1. The relation $Y^{r+1}=T\left(Y^{r}\right)$ has a unique exact solution $Y^{*} \in R$. 
2. For any initial solution $Y^{0} \in R$ the sequence defined in Equation (16)converges to $Y^{*}$.

\section{Proof}

1. Suppose that $T$ is a contraction mapping on $R$. Then $T$ is Lipchitz function by Theorem 2 and $T$ is continuous function by Theorem 3. Since $Y$ is exact solution, then

$$
Y^{*}=T\left(Y^{*}\right)
$$

is continuous on the region $R$. Now we want to show that, the equation (17) has a unique solution.

By contradiction, suppose that there exists another solution $Z^{*}$,such that $Z^{*} \neq Y^{*}$ which satisfy

$$
Y^{*}=T\left(Y^{*}\right) \text { and } Z^{*}=T\left(Z^{*}\right)
$$

Now

$$
\left|Y^{*}-Z^{*}\right|=\left|T\left(Y^{*}\right)-T\left(Z^{*}\right)\right| \leq\left|Y^{*}-Z^{*}\right| L,
$$

because $T$ is Lipchitz function. Then we get

$$
\left|Y^{*}-Z^{*}\right| \leq\left|Y^{*}-Z^{*}\right| L
$$

Hence $L \geq 1$ which is a contradiction to the definition of the Lipchitz constant, because $L<1$. Since $T$ is contraction mapping on the region $R$, then $Z^{*}=Y^{*}$. Therefore, Equation (16) has a unique solution.

2. By part one of this theorem we give the closure condition. That's mean If $Y^{0} \in R$ then $Y^{d} \in R$. Now $\left|Y^{d}-Y^{*}\right| \leq\left|T\left(Y^{d}\right)-T\left(Y^{*}\right)\right| \leq\left|Y^{d-1}-Y^{*}\right| L$, also $\left|Y^{d-1}-Y^{*}\right| \leq\left|T\left(Y^{d-1}\right)-T\left(Y^{*}\right)\right| \leq\left|Y^{d-2}-Y^{*}\right| L^{2}$, and so on, after $d$-times we get

$$
\left|Y^{d}-Y^{*}\right| \leq\left|Y^{d-(d-1)}-Y^{*}\right| L^{d-1} \leq\left|Y^{d-(d-1)-1}-Y^{*}\right| L^{d} \leq\left|Y^{0}-Y^{*}\right| L^{d},
$$

Take the limit of both sides we get

$$
\lim _{d \rightarrow \infty}\left|Y^{d}-Y^{*}\right| \leq \lim _{d \rightarrow \infty}\left|Y^{0}-Y^{*}\right| L^{d} .
$$

Since $0<L<1$, if $d \rightarrow \infty$ then $L^{d} \rightarrow 0$. That means $Y^{d} \rightarrow Y^{*}$ for $d \rightarrow \infty$. Hence, the sequence defined in equation (16) converges to $Y^{*}$.

\section{Conclusion}

In this work, we propose two methods called fixed point method and improve fixed point method to solve SVFI-2. Several numerical examples were tested using algorithms for FPM and IFPM for solving a system SVFI-2. The results given in Tables 1, 2, 3 and 4 , indicate clearly that both methods achieve good convergence as $r$ increases when the error decreases. The mentioned examples demonstrated the validity and applicability of the techniques. Finally, we concluded that IFPM converges faster than FPM as shown in Tables 5 and 6 . 
Table 5: Comparison between the absolute error of the present methods, FPM and IFPM methods in Example 1

\begin{tabular}{cllllll}
\hline \multirow{s}{*}{$s$} & & $\begin{array}{l}\text { Absolute } \\
\text { errors of } y_{1}(s) \\
\text { by FPM }\end{array}$ & $\begin{array}{l}\text { Absolute } \\
\text { errors of } y_{1}(s) \\
\text { by IFPM }\end{array}$ & $\begin{array}{l}\text { Absolute } \\
\text { errors of } y_{2}(s) \\
\text { by FPM }\end{array}$ & $\begin{array}{l}\text { Absolute } \\
\text { errors of } y_{2}(s) \\
\text { by IFPM }\end{array}$ & $\begin{array}{l}\text { Running } \\
\text { Time } \\
\text { second }\end{array}$ \\
\hline \multirow{3}{*}{0.2} & 0 & $1.1986 \times 10^{-1}$ & $1.9866 \times 10^{-1}$ & $4.5969 \times 10^{-1}$ & $4.5969 \times 10^{-1}$ & 0.019985 \\
& 2 & $4.5969 \times 10^{-2}$ & $5.5198 \times 10^{-3}$ & $5.7462 \times 10^{-2}$ & $2.0541 \times 10^{-2}$ & 0.083763 \\
& 4 & $5.7462 \times 10^{-3}$ & $2.6974 \times 10^{-3}$ & $7.1827 \times 10^{-3}$ & $3.7957 \times 10^{-3}$ & 0.165676 \\
& 6 & $7.1827 \times 10^{-4}$ & $2.0342 \times 10^{-4}$ & $8.9784 \times 10^{-4}$ & $6.1606 \times 10^{-4}$ & 0.331369 \\
& 8 & $2.1212 \times 10^{-5}$ & $1.3549 \times 10^{-5}$ & $2.2346 \times 10^{-4}$ & $1.0946 \times 10^{-5}$ & 0.388837 \\
& 10 & $4.3245 \times 10^{-6}$ & $3.9434 \times 10^{-6}$ & $3.3134 \times 10^{-5}$ & $6.6574 \times 10^{-6}$ & 0.477969 \\
& 12 & $6.2156 \times 10^{-7}$ & $1.8462 \times 10^{-7}$ & $3.2355 \times 10^{-6}$ & $7.6728 \times 10^{-7}$ & 0.585971 \\
& 14 & $1.2678 \times 10^{-7}$ & $1.0472 \times 10^{-8}$ & $5.7762 \times 10^{-7}$ & $1.0471 \times 10^{-8}$ & 0.655434 \\
16 & $6.7693 \times 10^{-8}$ & $5.3205 \times 10^{-9}$ & $2.2759 \times 10^{-7}$ & $1.0574 \times 10^{-9}$ & 0.754577 \\
& 18 & $8.4678 \times 10^{-9}$ & $1.0570 \times 10^{-10}$ & $4.6793 \times 10^{-8}$ & $8.7082 \times 10^{-10}$ & 0.848303 \\
& 20 & $2.4468 \times 10^{-9}$ & $5.6451 \times 10^{-11}$ & $5.6784 \times 10^{-9}$ & $4.9588 \times 10^{-11}$ & 0.961526 \\
\hline
\end{tabular}

Table 6: Comparison between the absolute error of the present methods, FPM and IFPM methods in Example 2

\begin{tabular}{cllllll}
\hline & & $\begin{array}{l}\text { Absolute } \\
\text { errors of } y_{1}(s) \\
\text { by FPM }\end{array}$ & $\begin{array}{l}\text { Absolute } \\
\text { errors of } y_{1}(s) \\
\text { by IFPM }\end{array}$ & $\begin{array}{l}\text { Absolute } \\
\text { errors of } y_{2}(s) \\
\text { by FPM }\end{array}$ & $\begin{array}{l}\text { Absolute } \\
\text { errors of } y_{2}(s) \\
\text { by IFPM }\end{array}$ & $\begin{array}{l}\text { Running } \\
\text { Time by } \\
\text { second }\end{array}$ \\
\hline \multirow{3}{*}{0.2} & 0 & $2.1402 \times 10^{-2}$ & $2.1402 \times 10^{-2}$ & $2.4280 \times 10^{-1}$ & $2.4280 \times 10^{-2}$ & 0.017213 \\
& 2 & $1.0652 \times 10^{-2}$ & $2.3705 \times 10^{-4}$ & $1.1583 \times 10^{-2}$ & $7.9818 \times 10^{-3}$ & 0.097263 \\
& 4 & $6.2613 \times 10^{-5}$ & $7.4575 \times 10^{-5}$ & $4.2278 \times 10^{-5}$ & $7.8007 \times 10^{-5}$ & 0.203957 \\
& 6 & $7.9443 \times 10^{-6}$ & $7.7778 \times 10^{-7}$ & $4.6111 \times 10^{-6}$ & $1.7745 \times 10^{-7}$ & 0.347614 \\
& 8 & $5.6095 \times 10^{-7}$ & $4.8578 \times 10^{-9}$ & $5.5970 \times 10^{-7}$ & $2.3335 \times 10^{-8}$ & 0.513815 \\
& 10 & $5.8513 \times 10^{-8}$ & $4.3890 \times 10^{-10}$ & $5.0802 \times 10^{-9}$ & $4.1740 \times 10^{-9}$ & 0.722167 \\
& 12 & $1.5373 \times 10^{-10}$ & $7.2264 \times 10^{-11}$ & $6.2804 \times 10^{-10}$ & $6.8422 \times 10^{-11}$ & 0.964843 \\
& 14 & $3.5835 \times 10^{-11}$ & $2.3652 \times 10^{-12}$ & $3.2524 \times 10^{-11}$ & $6.2924 \times 10^{-13}$ & 1.230646 \\
16 & $8.5912 \times 10^{-12}$ & $3.0622 \times 10^{-13}$ & $7.7321 \times 10^{-13}$ & $3.0642 \times 10^{-14}$ & 1.547392 \\
& 18 & $3.0213 \times 10^{-14}$ & $9.2433 \times 10^{-15}$ & $1.6325 \times 10^{-14}$ & $8.2420 \times 10^{-15}$ & 1.877719 \\
20 & $3.4421 \times 10^{-15}$ & $1.6952 \times 10^{-17}$ & $2.5534 \times 10^{-15}$ & $1.8732 \times 10^{-17}$ & 2.237260 \\
\hline
\end{tabular}




\section{Acknowledgments}

The authors would like to extend their sincere thanks to the referees for their helpful remarks and suggestions. Many thanks are due to the Salahaddin University and Universiti Teknologi Malaysia for providing the financial support for this research. The authors would also like to thank the Malaysian Ministry of Higher Education for providing financial support in FRGS grand number 4F625 for this research.

\section{References}

[1] Javadi, S. A. Modification in successive approximation method for solving nonlinear Volterra Hammerstein integral equations of the second. J. Mathematical Extension. 2014. 8(1): 69-84.

[2] Mathwalu, M. S. and Sulaiman, B. Numerical solution of second kind linear Fredholm integral equations using QSGS iteration method with high-order Newton-Cotes quadrature schemes. Malaysia J. Mathematical Science. 2011. 1(5) : 85-100.

[3] Maleknejad, K. Nouri K. and Torkzandeh, L. Comparison projection method for solving system integral equations. J. Bulletin of the Malaysian Mathematical Science Society. 2011. 2(34): 379-388.

[4] Yang, C. On the numerical solution of Volterra-Fredholm integral equations with Abel kernel using Legendre Polynomials. International Journal Advance Science and Technical Research. 2013. 3(1): 404-412.

[5] Mirzaee, F. and Hoseini, A. Numerical solution of nonlinear Volterra-Fredholm integral equations using hybrid of block-pulse functions and Taylor series. Journal of Alexandria Engineering. 2013. 3 (52): 551-555.

[6] Hasan, T. I. Salleh, S. B. and Sulaiman, N.A. Solving a System of Volterra-Fredholm Integral Equation of the second kind via series solution method. Journal of Applied Mathematic Sciences. 2014. 8(44): 2181-2194.

[7] Chniti, C. and Hou, J. Numerical method for solving non Volterra integral equations with convolution kernel. IAENG International Journal Applied Mathematics. 2002. $3(3)$ : $43-48$.

[8] Abdou, M. A. Volterra- Fredholm integral equation of first kind and contact problem. J. Applied mathematics and computation. 2012. 25(8): 177-193.

[9] Jerri, A. J. Introduction to Integral Equation with Applications. New York and Basel: Marcel Dekker, Inc. 1985.

[10] Sulaiman, N. A. Fixed point method and its improvement to solving Second kind Fredholm integral equation. J. Dohuk University. 2004. 7(1): 56-59.

[11] Sulaiman, N. A. and Hassan, T.I. Solution of a certain system of Fredholm integral equations of the first kind with symmetric kernels. J. Koya University. 2009. 13: 3-16 
[12] Waz Waz, A. M. Linear and Non-linear Integral Equations Methods and Applications. Berlin: Springer-Verlag. 2004.

[13] Codington, E. A. An Introduction to Ordinary Differential Equations. Third edition New Jersey: Prentice Hall 1961.

[14] Paripour, M. and Kamyar, M. Numerical solution of non-linear Volterra- Fredholm integral equations by using new basis functions. J. Communications in Numerical Analysis. 2013. 1(17): 1-12.

[15] Zarebnia, M. A numerical method of non-linear Volterra- Fredholm integral equations. Journal of Applied Analysis and Computation. 2013. 3: 95-104. 